\title{
On the Possibility of Cyclotron Maser Cooling
}

\author{
V. V. Parkhomchuk \\ Budker Institute of Nuclear Physics, Siberian Branch, Russian Academy of Sciences, \\ pr. Akademika Lavrent'eva 11, Novosibirsk, 630090 Russia \\ Received November 19, 2007; in final form, December 10, 2007
}

PACS numbers: 29.27.Bd

DOI: $10.1134 / \mathrm{S} 0021364008030132$

The use of the coherent (collective) radiation of a bunch to accelerate cooling in a magnetic field was discussed in [1]. The radiation power from an individual electron rotating in a circle of radius $\rho_{l}=m V_{\perp} c / e B$ in the magnetic field $B$ is given by the classical formula [2]

$$
P=\frac{2}{3 c^{3}}(\ddot{d})^{2}=\frac{4 e^{4} B^{2}}{3 m^{3} c^{5}} \frac{m V_{\perp}^{2}}{2}=\lambda E_{k},
$$

where $\lambda$ is the cooling decrement. The cooling time $\tau=$ $1 / \lambda$ in normal fields, $B=1 \mathrm{kG}$, is equal to about $257 \mathrm{~s}$. Berezovskii and Men'shikov [1] proposed grouping electrons in a coherent bunch, which allows an increase in the oscillating dipole moment $d$ by a factor of the number of particles, $N_{e}, d=e N_{e} \rho_{l}=N_{e} m c V_{\perp} / B$. In this case, the radiation power increases by $N_{e}^{2}$ and the cooling decrement increases by $N_{e}$, leading to the ultrafast cooling of electrons in a time of about $10^{-6} \mathrm{~s}$ for $N_{e}=$ $10^{8}$. In reality, in the absence of the correlation between the electrons, the dipole moment is determined by statistical fluctuations $d_{s}=e \sqrt{N_{e}} \rho_{l}$ and the radiation power is the sum of radiation from all of the particles. According to Liouville's theorem, the frequency spread described by Eq. (5) in [1] leads to the statistical mixing rather than to bunching, as thought by the authors. For bunching, it is necessary to ensure the interaction with resonators so that the thermal motion does not mix par- ticles, but the thermal energy after the development of the maser instability increases noticeably due to the nonadiabaticity of bunching, rather than decreases. Such "brilliant" prospects have already attracted "hothead" researchers [3] and a special section was organized for the discussion of cyclotron maser cooling in 1993 in the workshop on beam cooling and related topics [4]. As said by van der Meer, a Nobel Prize winner, "cyclotron maser cooling is stated as ultrafast, the cooling time is only several turns. However, the coherent radiation does not contain information on the motion of individual particles. For this reason, cyclotron maser cooling will not work."

\section{REFERENCES}

1. V. V. Berezovskii and L. I. Men'shikov, Pis'ma Zh. Éksp. Teor. Fiz. 86, 411 (2007) [JETP Lett. 86, 355 (2007)].

2. L. D. Landau and E. M. Lifshitz, Course of Theoretical Physics, Vol. 2: The Classical Theory of Fields (7th Rus. ed., Nauka, Moscow, 1988, p. 256; 4th Eng. ed., Butterworth-Heinemann, 1980).

3. H. Ikegami, Phys. Rev. Lett. 64, 1773, 2593 (1990).

4. S. van der Meer, in Proceedings of Workshop on Beam Cooling and Related Topics, Montreux, October 4-8 (1993), p. 123; CERN 94-03, April 26, 1994, Proton Synchrotron Division. 